\title{
Factors associated with sensitive regression weights: A fungible parameter approach
}

\author{
Robert A. Agler ${ }^{1,2}$ (I) Paul De Boeck ${ }^{2,3}$
}

Published online: 3 June 2019

(C) The Psychonomic Society, Inc. 2019

\begin{abstract}
Sensitive parameters serve as a weak foundation for scientific inferences, because they provide less certainty about the accuracy and trustworthiness of the estimated model. Fungible weights may be used to examine parameter sensitivity by looking at how much sets of interchangeable, slightly suboptimal linear regression weights, all of which yield an identical, slightly reduced value of $R^{2}$, differ from the optimal OLS weights. We find that in the two-predictor case, the range of a predictor's fungible weights is almost completely explained by the absolute value of the correlation of the other predictor with the criterion variable $\left(R^{2}=.990\right)$; an interaction with the variance inflation factor $(V I F)$ yields $R^{2}=1$. In the more complicated three-predictor case, the effects of the other two correlations yield $R^{2}=.839$, and including the predictor's $V I F$ and its interactions yields $R^{2}=.910$. The effects observed occur because alternative predictors with a high correlation with the criterion, or with each other, can compensate for the changes to a predictor's weight while still yielding similar predicted values. An R function is provided to calculate the range of fungible weights for a given covariance matrix. We close with a discussion of some important implications of our results regarding parameter sensitivity and the trustworthiness of effect estimates.
\end{abstract}

Keywords Fungible weights $\cdot$ Parameter sensitivity $\cdot$ Linear regression

Fungible weights are sets of alternative, suboptimal weights that may be used to examine parameter sensitivity in multiple linear regression models by way of a minor decrement in $R^{2}$. Each set of weights yields the same value of $R^{2}$, as well as the same correlation between the ordinary least squares (OLS) and alternative predicted values (Waller, 2008). The degree of discrepancy between sets of fungible weights and the OLS weights is independent of sample size, and their behavior is determined by factors other than those that determine the confidence interval for regression weights (Jones, 2013; Pek, Chalmers, \& Monette, 2016). Under some conditions, even very small decrements in $R^{2}$ can be associated with substantially different regression weights for the predictor variables.

Robert A. Agler

agler.24@osu.edu

1 College of Public Health, Division of Epidemiology, The Ohio State University, Columbus, OH, USA

2 Department of Psychology, The Ohio State University, Columbus, OH, USA

3 KU Leuven, Leuven, Belgium
In those cases, the weights are considered sensitive and provide a poor basis for scientific conclusions (Green, 1977), and it is therefore prudent to ensure that the weights obtained in a study are not too sensitive.

The uncertainty of parameter estimates we focus on here is not the uncertainty that stems from sampling variation, but uncertainty stemming from possible model inaccuracies, which are rarely known in practice. Although the two types of uncertainty are related (Pek et al., 2016), tests for one cannot serve as tests for the other. Methods of examining parameter sensitivity can be understood as relaxing the degree of certainty about the model's accuracy (rather than relaxing the degree of certainty about precision, as with standard errors and confidence intervals) and as an acknowledgment that all models are wrong and almost necessarily biased (Box, 1976; Edwards, 2013), due to, for instance, missing variables, incorrect error terms, or missing (interaction) terms, or because measurement error is ignored (Jaccard \& Wan, 1995). Although the sources of bias may cancel each other out to some degree, in general it can be expected that more inaccurate models will also be more biased. Here we will make use of predictor-specific fungible weight intervals (FIs) to indicate the sensitivity of parameter estimates to possible model 
inaccuracy, in analogy to how confidence intervals (CIs) reflect the imprecision stemming from sampling variation. The two sources of uncertainty are different, and as will be shown, the two types of intervals are different, as well.

Before continuing, we wish to make clear that weights that yield a lower value of $R^{2}$ are not necessarily less accurate. On the contrary, the accurate weights will yield a lower value of $R^{2}$ if the model is incorrect. The estimated parameters in a linear regression model are optimal weights given the data and the model, but these estimates are unlikely to reflect the true effects associated with each variable if the model is not completely accurate (i.e., if it is not the "true model"). Examining the weights associated with a decrement in $R^{2}$ obviously does not guarantee recovery of the true effects, but it nonetheless provides an opportunity to investigate the potential consequences of an inaccurate model on the regression effect estimates, without being limited to any specific type of model inaccuracy.

Suppose that a model $A$ is an accurate model and model $B$ is an inaccurate model that does not include one or more predictors. If one were to use the true parameter values from model $A$ in the same model $B$, the result would be a value of $R^{2}$ lower than that obtained with OLS estimation, and the accurate, unbiased parameters from model $A$ would seem inferior to the OLS estimates. For example, suppose that the true model $A$ includes a set of four predictor variables and a criterion variable with all $r \mathrm{~s}$ $=.4$, and so all $\beta \mathrm{s}=.182$ and $R^{2}=.291$. If a predictor is excluded in the inaccurate model $B$, then the OLS estimates for the remaining three predictors would all be $\beta \mathrm{s}=.222$ and $R^{2}=.267$. However, if the true regression weights from model $A$ were used in model $B$, then $R^{2}=.218$, which is clearly inferior to the .267 value obtained with OLS. Similarly, when the true model $A$ is a model with five predictor variables and all $r \mathrm{~s}=.4$, then $R^{2}=.308$ and all $\beta \mathrm{s}=.154$. However, if only three of the five predictors are used in a model $B$, the OLS estimates are again $\beta \mathrm{s}=.222$ and $R^{2}=.267$, whereas the correct values (from model $A$ ) used in model $B$ yield an even larger reduction, $R^{2}=.185$. Counterintuitively, then, optimal weights are not necessarily correct weights. Whereas these examples are calculated working with given true weights and known violations in an incorrect model, in practice the calculation of the sensitivity of weights is a form of reverse calculation that does not guarantee recovery of the true weights unless the specific violations are known (and we do not claim here that fungible weights may be used to recover the true model, as there has been insufficient research to make such a claim). Instead, consideration of alternative weights provides a general indication of how much the obtained parameters may reflect bias due to model violations of any form.

\section{Fungible regression weights}

Fungible regression weights are alternative weights that yield predicted values for the criterion variable that yield a prespecified criterion correlation with the OLS predicted values and identical suboptimal values of $R^{2}$ - thus, the term fungible weights. We denote the OLS vector of weights as $\boldsymbol{b}$, and the vector of alternative weights as $\boldsymbol{a}$. Similarly, we denote the OLS value of $R^{2}$ as $R_{b}^{2}$ and the fungible value as $R_{a}^{2}$, the predicted OLS weight values as $\hat{y}_{b}$, the predicted fungible weight values as $\hat{y}_{a}$, and the prespecified correlation between the two as $r_{\hat{y}_{a} \hat{y}_{b}}$. For two predictor variables, there are two sets of alternative weights that satisfy the constraints, but for three or more predictors, there are an infinite number of alternative weights that do so (fungible weights are not defined for a single predictor). The full mathematical derivation of fungible weights may be found in Waller (2008), and a means to identify the minimum and maximally discrepant weight sets (i.e., the fungible extrema) may be found in Waller and Jones (2009). Fungible weights for logistic regression may be found in Jones and Waller (2016), and Lee, MacCallum, and Browne (2018) developed fungible parameters for structural equation modeling. Alternative weights that yield the same value of $R_{a}^{2}$ but do not satisfy the prespecified correlation are known as exchangeable weights (Pek et al., 2016).

Although we wish to minimize the use of equations throughout the present article, to reach a broader audience, a brief explanation of the geometry of fungible weights will be useful for the discussion of our results. Geometrically, fungible weight sets with three or more predictors lie at the intersection of a $p-1$ dimensional (hyper) plane and a $p$-dimensional (hyper)ellipsoid. The intersection is a $p-1$ dimensional ellipse or (hyper)ellipsoid. With two predictors, the weights sets are the two points at which a line intersects with an ellipse. The intersecting ellipse emerges because each weight is a tightly constrained function of the others, because of the prespecified correlation $r_{\hat{y}_{a} \hat{y}_{b}}$ (Waller \& Jones, 2009). The (hyper) plane is characterized by the set of weight vectors that satisfy the constraint that they yield the same value of $R_{a}^{2}$ - specifically,

$R_{a}^{2}=\boldsymbol{a}^{\prime} \boldsymbol{R}_{\boldsymbol{x x}} \boldsymbol{b}$

and the (hyper) ellipsoid is characterized by the set of weight vectors that satisfy

$R_{a}^{2}=\boldsymbol{a}^{\prime} \boldsymbol{R}_{\boldsymbol{x x}} \boldsymbol{a}$

where $\boldsymbol{R}_{\boldsymbol{x} \boldsymbol{x}}$ is the predictor correlation matrix (Jones \& Waller, 2016; Waller \& Jones, 2009). Fungible weights are those that satisfy both equations.

We will refer to the ellipsoid as the all-possible-regressions ellipsoid, because for a given $R_{a}^{2}$ and predictor matrix, an infinite number of possible weight sets will yield the same $R_{a}^{2}$ (this is also true for $R_{b}^{2}$; Waller \& Jones, 2011). Parameter sensitivity is evaluated on the basis of how tight the set of weights are around the parameters associated with optimal 
model fit, with tighter sets of weights providing a stronger basis for inferences from the OLS estimates. In other words, smaller ellipses indicate less sensitive parameters.

An example of fungible weights is shown in Fig. 1 with three predictor variables, with the variance explained for the OLS estimates being $R_{b}^{2}=.647$. Given $r_{\hat{y}_{a} \hat{y}_{b}}=.90, .95$, and .99 , the resultant alternative values are $R_{a}^{2}=.524, .584$, and .634 , respectively. The single dot represents the OLS estimates, and the ellipses represent the fungible weight sets for a given $r_{\hat{y}_{a} \hat{y}_{b}}$ value. As can be seen, there are large discrepancies between the OLS estimates and some of the fungible weights. In the case of $\beta_{X_{1}}$, the OLS weight is .073 and is significant for $N=$ 450 , whereas the fungible weights include both positive and negative weights for all shown values of $r_{\hat{y}_{a} \hat{y}_{b}}$. For $\beta_{X_{2}}$, the OLS weight is .239 and is significant for samples as small as $N$ $=50$, yet for $r_{\hat{y}_{a} \hat{y}_{b}}=.90$, the fungible weight sets also include negative weights.

Although it is easy to see that the sizes of the fungible weight ellipses differ for each predictor in this example, it is not immediately apparent what factors other than the arbitrary value of $r_{\hat{y}_{a} \hat{y}_{b}}$ might contribute to the fungible ellipses. Several factors that affect fungible weights are detailed in Jones (2013). We distinguish these factors as being related to the predictor correlation matrix, to the predictor-criterion correlation vector, or to both. Factors related to the predictor correlation matrix may be understood as a matter of multicollinearity. Specifically, the

\section{Fungible Weight Sets}

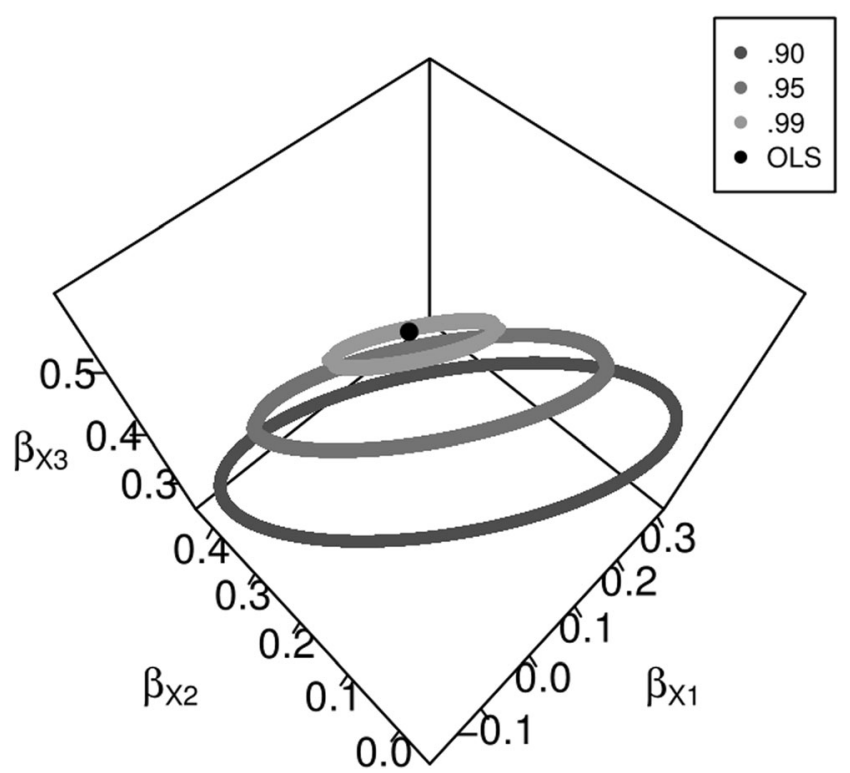

Fig. 1 Fungible weights based on the case that $r_{X_{1} X_{2}}=.1, r_{X_{1} X_{3}}=.2$, $r_{X_{2} X_{3}}=.3, r_{X_{1} Y}=.2, r_{X_{2} Y}=.4$, and $r_{X_{3} Y}=.6$, with associated regression weights of $\beta_{X_{1}}=.073, \beta_{X_{2}}=.239$, and $\beta_{X_{3}}=.514$. The variance explained for the OLS estimates is $R_{b}^{2}=.647$, and $R_{a}^{2}=.524$, .584 , and .634 , for $r_{\hat{y}_{a} \hat{y}_{b}}=.90, .95$, and .99 , respectively eigenvalues of $\boldsymbol{R}_{\boldsymbol{x} x}$ determine the shape of the ellipsoid, and the orientation of this ellipsoid is determined by the eigenvectors. As multicollinearity increases, the first eigenvalue will increase and the last eigenvalue will decrease, approaching zero. Roughly equal eigenvalues yield an ellipsoid that is roughly (hyper) spheroidal, and fungible weights yield (hyper) ellipses that are more circular. In contrast, the more discrepant the eigenvalues are, the thinner the ellipsoid becomes in at least one dimension (e.g., cigar or pancake shaped). The axes of the ellipsoid are calculated as follows:

$l_{i}=2 \sqrt{\frac{R_{\alpha}^{2}}{\lambda_{i}}}$

where $l_{i}$ denotes the $i$ th axis length, and $\lambda_{i}$ denotes the $i$ th ordered eigenvalue of the predictor matrix (Waller \& Jones, 2011). It follows from the effects of the eigenvalues that measures of multicollinearity may help explain the fungible weight intervals without necessarily playing a direct role. The determinant is equal to the product of the eigenvalues of the matrix, so that as the discrepancy between the first and last eigenvalues increases, the determinant decreases because the last eigenvalue approaches zero. Similarly, the ratio between the first and last eigenvalues is known as the condition number, which is another measure of multicollinearity. Additionally, the familiar variance inflation factor $(V I F)$ tends to increase as the discrepancy between the eigenvalues increases.

Factors related to the predictor-criterion correlation vector may be understood as being due to the $r_{\hat{y}_{a} \hat{y}_{b}}$ constraint for the predicted values. The orientation of the eigenvectors of $\boldsymbol{R}_{\boldsymbol{x} x}$ with respect to the vector of standardized regression coefficients (i.e., the correlation between the two) plays a role because, as we previously mentioned, the eigenvectors determine the orientation of the ellipsoid, and the intersecting plane is defined by the set of weight vectors that satisfy $R_{a}^{2}=\boldsymbol{a}^{\prime} \boldsymbol{R}_{x x} \boldsymbol{b}$. As a result, where the plane intersects the ellipsoid is determined by both $\boldsymbol{b}$ and $\boldsymbol{R}_{x \boldsymbol{x}}$, with different intersection points being associated with different curvatures and thicknesses of the ellipsoid (Jones, 2013). Weight vectors more closely related to the eigenvectors will be more closely related to the variance of the predictors. Additionally, the angle of the intersecting plane is determined by the predictorcriterion correlations, because the correlation vector $\boldsymbol{r}_{\boldsymbol{x} y}$ is orthogonal to the (hyper) plane by design (Waller \& Jones, 2009), so the correlations can be expected to predict fungible weight behavior.

Finally, larger values of $R_{b}^{2}$ lead to larger ellipsoids composed of all weights that yield the same value of $R_{a}^{2}$, given the same value of $r_{\hat{y}_{a} \hat{y}_{b}}$. To understand this, consider that just as there are an infinite number of weight vectors that satisfy the equation for the (hyper) ellipsoid, $R_{a}^{2}=\boldsymbol{a}^{\prime} \boldsymbol{R}_{x x} \boldsymbol{a}$, there are also 
an infinite number of weight vectors that satisfy $R_{b}^{2}=\boldsymbol{b}^{\prime} \boldsymbol{R}_{\boldsymbol{x} x} \boldsymbol{b}$ (Waller \& Jones, 2011). As a result, as $R_{b}^{2}$ increases, so too must the absolute value of some components of either $\boldsymbol{R}_{\boldsymbol{x} x}$ or $\boldsymbol{b}$. The number of predictors also impacts the fungible ellipses indirectly, due to the possible predictor correlations being constrained by the number of predictors (Jones, 2013), but we do not consider this matter further here.

Here, rather than the intersection ellipse, we focus our investigation on the range of each weight separately. We will refer to the range as the fungible interval, a sort of "validity interval" analogous to the reliability interval provided by a confidence interval. We use the range per predictor for a few reasons. In addition to the ease of interpreting the difference between two values, it is also the case that the familiar confidence interval is a range of weight values per predictor. There is also heavy bimodality in the distribution of the fungible weights, with peaks near the boundaries (Waller, 2008), and as a result, the range implies relatively little loss of information. Finally, we focus on the range rather than the fungible extrema (Waller \& Jones, 2009) because the range is far more computationally simple. Fungible extrema are the two weight sets that either minimize or maximize the cosine (equivalently, the correlation) between the fungible and OLS weights. Though there is overlap between the minimally and maximally discrepant weight sets and the minimum and maximum weights for each predictor, the weight sets that include either the minimum or the maximum values of a weight are not necessarily fungible extrema, because there are $2 p^{2}$ weights for the range end points, given three or more predictors (where $p$ denotes the number of predictors), but only $2 p$ weights associated with the extrema.

Our purpose here, then, is to consider both how much the fungible interval can vary in size and what factors can help explain the differences, with the goal of providing a general sense of when the parameters may be sensitive to the effects of unknown model violations. Though the factors we explore are theoretically motivated, we take a relatively more applied approach in our reporting, with a preference for simple and familiar explanatory factors. We will work from correlation matrices in our studies, since they are always available to researchers, but knowledge of how the model is inaccurate is rarely so.

\section{Fungible intervals for the two-predictor case}

We begin with the two-predictor case for fungible intervals, because for two predictors there are only two weight sets. The two sets are the two end points of a line - that is, the fungible interval. In the next step we will consider the more complicated threedimensional, three-predictor case, from which we can draw some possible generalizations to even larger predictor sets.

\section{Method}

For this study, the possible correlations between the two predictor variables and the criterion variable were $-.5,-.4,-.3$, $-.2,-.1,0, .1, .2, .3, .4$, and $.5 .^{1}$ Because there are three correlations in a three-variable correlation matrix, this resulted in $11^{3}=1,331$ matrices. We follow the example of confidence intervals and use the following criterion values: $r_{\hat{y}_{a} \hat{y}_{b}}=.90$, .95 , and .99 . These criterion values result in relatively modest drops in variance explained. For example, for a value of $R_{b}^{2}=$ .25 , the resultant $R_{a}^{2}$ values would be $.203, .226$, and .245 , respectively.

For each matrix (all combinations here produce valid correlation matrices, although some-e.g., all $r \mathrm{~s}=-.5$ and $R_{b}^{2}=$ 1 - are extremely unlikely and may be computationally difficult in practice), we calculated the OLS weights and the two fungible weight pairs for each $r_{\hat{y}_{a} \hat{y}_{b}}$ value. All weights derived are standardized weights. Additionally, to provide some sense of the magnitude of the fungible intervals and provide a point of comparison, we also calculated $95 \%$ confidence intervals based on $N=100$. To calculate the two sets of alternative weights, we used the R function provided in Waller (2008), with a small modification to allow estimation with two predictors.

\section{Results and discussion}

Comparison with confidence intervals As with confidence levels and interval size, lower $r_{\hat{y}_{a}} \hat{y}_{b}$ values result in larger intervals than do higher $r_{\hat{y} a} \hat{y}_{b}$ values. These results are to be expected because, generally speaking, increasingly discrepant predictions necessitate increasingly discrepant variable weights. The minimum and maximum interval sizes for $\beta_{X_{1}}$ and $\beta_{X_{2}}$ were identical: For each criterion correlation value, the largest intervals were $0.453,0.343$, and 0.161 , for $r_{\hat{y}_{a} \hat{y}_{b}}$ values of $.90, .95$, and .99 , respectively, and the smallest intervals were equal to zero - that is, the fungible weights were equal to the OLS weights. For comparison, the $95 \%$ confidence intervals ranged in size from .179 to .465 . The magnitudes of each predictor's fungible intervals were perfectly correlated across all three criterion values.

Figure 2 shows the end points for the two types of intervals. Since the plot for $\beta_{X_{2}}$ is identical, we hereafter use the subscript $i$ to denote a specific predictor, and the subscript $i *$ to denote the other. The OLS point estimates and confidence interval end points are shown in black. The fungible interval end points are shown for $r_{\hat{y}_{a} \hat{y}_{b}}=.90$ and .99 , in dark and light gray, respectively. The end points for $r_{\hat{y}_{a} \hat{y}_{b}}=.90$ overlap with

\footnotetext{
$\overline{1}$ Technically, we do not use 0 but $1 * 10^{-10}$, both here and for the threepredictor case. We do so because the fungible function's calculation fails if all predictors have a zero correlation with the criterion variable.
} 
those for $r_{\hat{y}_{a} \hat{y}_{b}}=.99$. The end points for .95 lie between these two sets of end points, and so are not shown. Both intervals are symmetrical around a set of weights. Specifically, the confidence intervals are symmetrical about the OLS weights, whereas the fungible interval end points are symmetrical about the transformed OLS weights (not shown) that are at the center of the fungible ellipse. These transformed weights are equal to $\frac{R_{a}^{2}}{R_{b}^{2}} b$ (Waller \& Jones, 2009). Because these weights are necessarily closer to 0 than the OLS weights, so too are the fungible interval end points.

Figure 3 shows the magnitude of the intervals (the difference between the lowest and highest weights), plotted in relation to the OLS values of $\beta_{X_{i}}$. We use $r_{\hat{y}_{a} \hat{y}_{b}}=.99$ in the figure in order to minimize overlap between the confidence and fungible intervals; smaller criterion values simply result in increased distance between points without affecting the overall pattern. It is immediately apparent that the fungible and confidence interval magnitudes follow quite different patterns. Larger values of $\beta_{X_{i}}$ are generally associated with tighter confidence intervals, but the fungible interval magnitudes are almost completely unrelated to the value of $\beta_{X_{i}}$. Additionally, whereas the confidence intervals cluster in a singular arcing swath, the fungible intervals cluster in six groupings. Of note is that these clusters are not equally diffuse. From bottom to top, the first cluster includes entirely zero-magnitude intervals, and each subsequent cluster is increasingly diffuse.

\section{$\mathrm{Cl}$ and FI End Points}

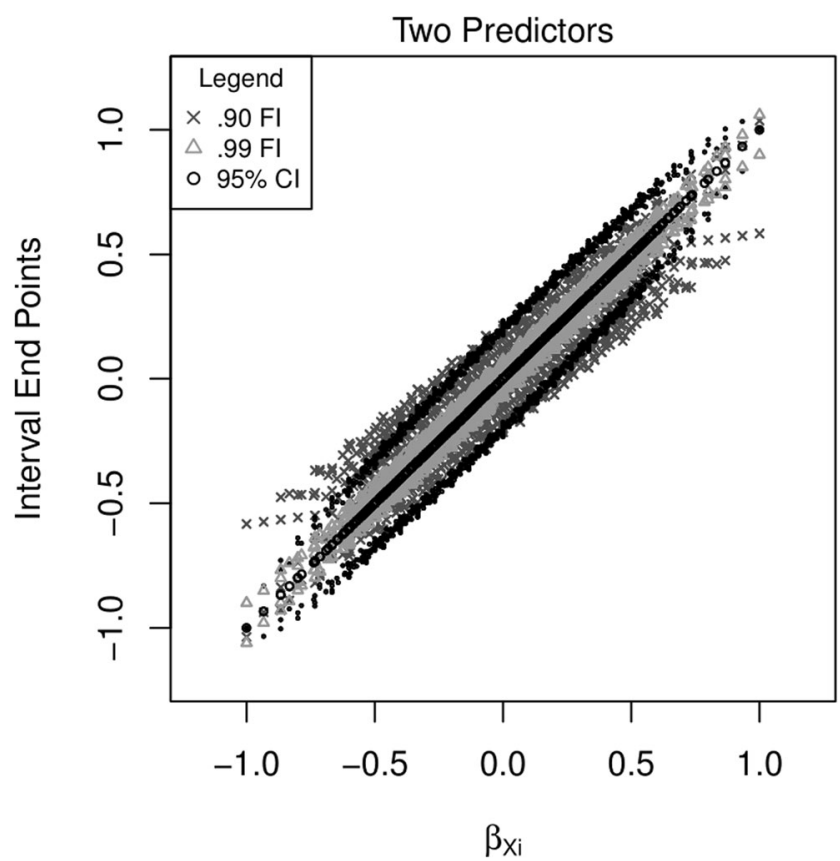

Fig. 2 End points of the confidence and fungible intervals for two predictors, plotted against the OLS point estimates. Confidence intervals are based on $N=100$. The values of $r_{\hat{y}_{\mathrm{a}}} \hat{\mathrm{y}}_{\mathrm{b}}$ used here are .90 (dark gray) and .99 (light gray). Some end points for $r_{\hat{y}_{\mathrm{a}} \hat{y}_{\mathrm{b}}}=.90$ are overlapped by the points for $r_{\hat{\mathrm{y}} \mathrm{a}} \hat{\mathrm{y}}_{\mathrm{b}}=.99$

\section{$\mathrm{Cl}$ and $\mathrm{FI}$ Interval Magnitude Two Predictors}

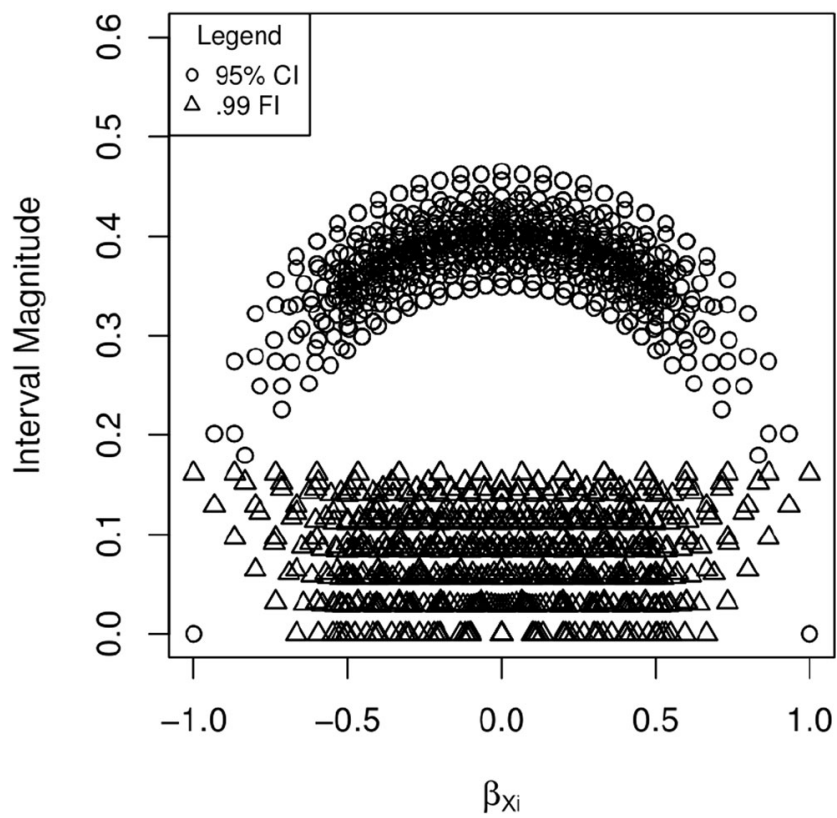

Fig. 3 Fungible interval (FI) $\left(r_{\hat{y}_{\mathrm{a}} \hat{y}_{\mathrm{b}}}=.99\right)$ and $95 \%$ confidence interval (CI) magnitudes plotted against the value of $\beta_{X_{i}}$. Confidence intervals are based on $N=100$

Considering the conditions in this study, this suggests that the absolute magnitude of one of the correlations may be the primary predictor of the fungible interval size, and that there is an interaction between it and at least one other variable.

Explanatory factors for fungible interval size Because we are using regressions to describe regressions, using the same regression terminology (e.g., predictor and criterion or (in) dependent variables) for both can easily become confusing. For the regressions associated with fungible weights, we use the predictor and criterion terminology to refer to the variables involved, and for the regressions to explain the fungible interval size, we use the terms explanatory and explained variable(s).

We considered the following explanatory variables, in both pairs and trios, with all interactions included: $\left|r_{X_{i} Y}\right|,\left|r_{X_{i^{*}} Y}\right|$, $\left|r_{X_{1} X_{2}}\right| ; V I F$ (there is only one value with two predictors); the determinant, condition number, and eigenvalues of the predictor matrix; the OLS regression weights; and $R_{b}^{2}$. We also considered the correlations (direction cosines) between the predictor matrix eigenvectors and the OLS weight vectors, and the axes of the ellipse (what would be the all-possibleregressions ellipsoid for three predictors). The explanatory variable weights we report are for standardized explanatory variables, but the explained variable - the fungible intervalis not standardized. We take this approach because standardization of the explanatory variables eases interpretation of the coefficients by making them comparable within the same 
analysis, but standardization of the explained variable would mask differences in the widths of the fungible intervals associated with values of $r_{\hat{y}_{a} \hat{y}_{b}}$. All weights are exact, since no sampling was involved in this study (the confidence intervals were calculated directly from the standard errors based on correlation matrices and the assumed $N=100$ )

In contrast to the complicated, multifactorial determination of the shape and orientation of the (hyper) ellipsoid and orientation of the intersecting (hyper) plane (Jones, 2013) for three or more predictors, we found that the range for a given predictor's fungible interval in the two-predictor case is very simply determined. As can be seen in Table 1 , the magnitude of the $\beta_{X_{i}}$ fungible interval is almost completely explained by $\left|r_{X i * Y}\right|$. This single variable is sufficient to yield $R_{b}^{2}=.990$. By including $V I F$ in the regression, the result is a model with $R_{b}^{2}=$ .997; all weights in these models were positive. With the addition of the interaction term, the result is $R_{b}^{2}=1$, so these three terms perfectly explain the range for the twopredictor case. We also briefly note that the combination of $\left|r_{X_{i^{*}} Y}\right|$ and the second axis of the ellipse resulted in $R_{b}^{2}$ $=.992$. We will revisit this point in the three-predictor case. Figure 4 illustrates the relationship between $\left|r_{X^{*} Y}\right|$ and the range of $\beta_{X_{i}}$, with the magnitude of the fungible interval increasing with values of $\left|r_{X_{i^{*}} Y}\right|$. The increasing spread reflects the interaction between $\left|r_{X_{i *} Y}\right|$ and VIF.

The results presented here may be understood by considering that satisfying the $r_{\hat{y}_{a} \hat{y}_{b}}$ constraint requires that when the weight of one predictor goes up, the weights of the others go down. This is perfectly the case for two predictors, and thus for the two pairs of fungible weights, but for more predictors, it follows from the elliptical form. The results in Fig. 4 show that the fungible interval for the weight of one predictor increases with the

Table 1 Regression weights of explanatory variables for fungible intervals in the two-predictor case

\begin{tabular}{lllll}
\hline & \multicolumn{1}{l}{$r_{\hat{y}_{a} \hat{y}_{b}}$} & & & \\
\cline { 2 - 5 } & .90 & .95 & .99 & $\Delta R_{b}^{2}$ \\
\hline Intercept & 0.226 & 0.171 & 0.081 & \\
$\left|r_{X_{i^{*} Y}}\right|$ & 0.133 & 0.101 & 0.047 & .990 \\
$V I F$ & 0.012 & 0.009 & 0.004 & .007 \\
$\left|r_{X_{i^{*} Y}}\right| \times V I F$ & 0.007 & 0.005 & 0.002 & .003 \\
& & & & $R_{b}^{2}=1$ \\
\hline
\end{tabular}

Values of $\Delta R_{b}^{2}$ indicate the increase in $R_{b}^{2}$ associated with each term being added to separate regression models. Coefficient values are identical for both predictors. The regression weights are smaller in the columns farther to the right because the fungible intervals are smaller.

\section{Fungible Interval Magnitude Two Predictors}

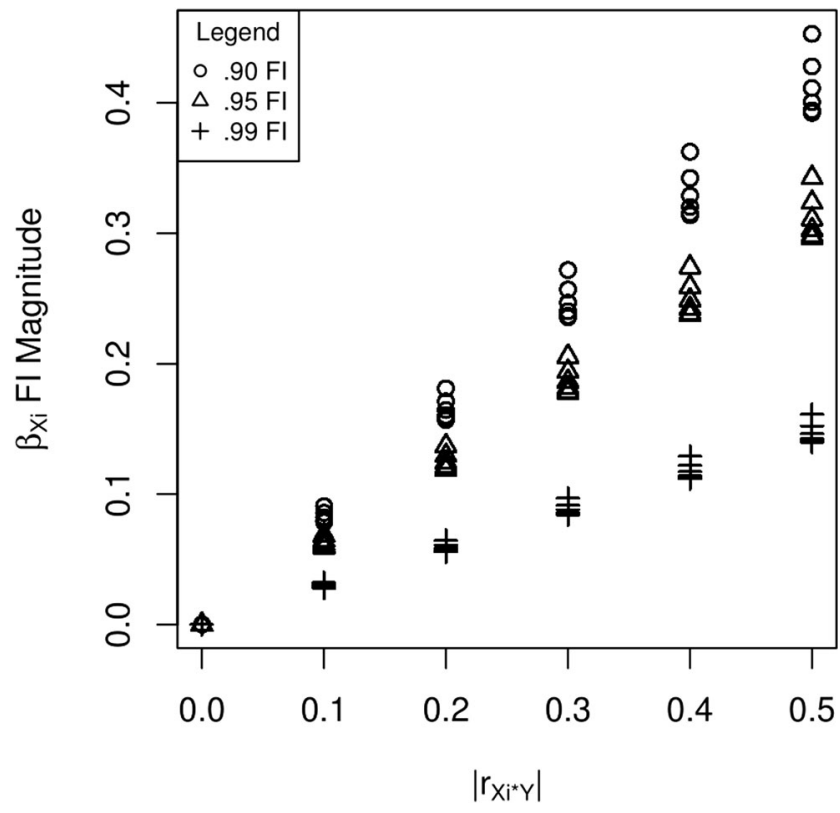

Fig. 4 Magnitudes of the $\beta_{X_{i}}$ fungible intervals in the two-predictor case, plotted against $\left|r_{X i * Y}\right|$. The increased spread of the interval magnitudes as $\left|r_{X i * Y}\right|$ increases is because of its interaction with VIF

correlation between the other predictor and the criterion variable, and slightly more so if VIF is high. Varying the weights of a predictor has fewer consequences for the prediction when the other variable is highly correlated with the criterion variable, and can therefore compensate for the weight changes, so there is more freedom for the weight to move. Consistent with this, when $\left|r_{X i * Y}\right|$ is equal to 0 , then the magnitude of the fungible interval for $\left|r_{X i Y}\right|$ is also equal to 0 . Additionally, the intersecting plane is, by design, orthogonal to the predictor-criterion correlation vector (Waller \& Jones, 2009 , p. 594). In other words, the angle of the plane and the resultant intersection ellipse are entirely determined by the predictor-criterion correlations. It follows that if the correlation of one of the two predictors with the criterion is zero, then the fungible interval for the other predictor is zero, as can be seen in Fig. 1 of Waller and Jones (2009, p. 592). Our finding that the fungible interval shrinks to zero if the other correlation is zero confirms the geometric analysis in Waller and Jones (2009).

When $X_{1}$ and $X_{2}$ are highly correlated (high VIF), then the range is somewhat larger, because then one weight can better compensate for the other. The higher the correlation between the two predictors, the less it matters if one weight is increased at the cost of another, but this compensation is far from the 
primary factor determining the interval size. If $X_{1}$ is highly related to $Y$ and $X_{2}$ is not related, then decreasing $\beta_{X_{1}}$ and increasing $\beta_{X_{2}}$ will have a large detrimental effect, because giving $X_{2}$ a larger weight adds noise to the prediction, leaving almost no freedom for $\beta_{X_{1}}$ to change while still satisfying $r_{\hat{y}_{a} \hat{y}_{b}}$, regardless of multicollinearity. The reverse is true if $X_{2}$ is highly correlated with $Y$ but $X_{1}$ is not: Decreasing the value of $\beta_{X_{2}}$ and increasing the value of $\beta_{X_{1}}$ will, for the most part, simply add noise. It appears, then, that if there is a good alternative predictor, it does not matter too much which one does the predictive work, and this is slightly more the case if the two predictors are highly correlated.

\section{Fungible weights with three predictors}

\section{Method}

For this study, the possible values for $r_{\hat{y}_{\mathrm{a}} \hat{y}_{\mathrm{b}}}$ were again. $90, .95$, and .99. We limited ourselves to $r \mathrm{~s}=-.5,-.3,-.1,0, .1, .3$, and .5 to make the number of conditions computationally manageable. In a four-variable system there are six correlations, so in this case, with seven possible values, $7^{6}=117,649$ matrices were generated. Of these, 109,129 were valid correlation matrices, and for each we derived the OLS regression weights and confidence intervals based on $N=100$, and calculated 1,000 fungible weight trios using Waller's (2008) R function. These 1,000 sets were sufficient to recover the shape of the fungible weight ellipse as well as any trends, and there was only a minor loss in precision.

\section{Results and discussion}

Comparison with confidence intervals Figure 5 shows the end points for both the confidence and fungible intervals with three predictors, plotted in relation to $\beta_{X_{1}}$ (the plots for $\beta_{X_{2}}$ and $\beta_{X_{3}}$ are identical). As in the two-predictor case, the confidence intervals are symmetric about the OLS point estimates, and the fungible intervals are symmetric about the $\frac{R_{a}^{2}}{R_{b}^{2}}$ $b$ transformed weights. The OLS point estimates and confidence interval end points are shown in black. The fungible interval end points are those for $r_{\hat{y}_{a} \hat{y}_{b}}=.90$ and .99 and are again shown with dark and light gray, respectively. The end points for $r_{\hat{y}_{a} \hat{y}_{b}}=.90$ overlap with those for $r_{\hat{y}_{a} \hat{y}_{b}}=.99$, and we again do not show the points for $r_{\hat{y}_{a} \hat{y}_{b}}=.95$ because they are between the two criterion values shown. Unlike in the previous study with two predictors, here the points for $r_{\hat{y}_{a} \hat{y}_{b}}=.99$ overlap with the $95 \%$ confidence interval end points.

Figure 6 shows the magnitude of the fungible intervals (the difference between the lowest and highest bounds) plotted in relation to $\beta_{X_{1}}$ (the plots for $\beta_{X_{2}}$

\section{$\mathrm{Cl}$ and FI End Points}

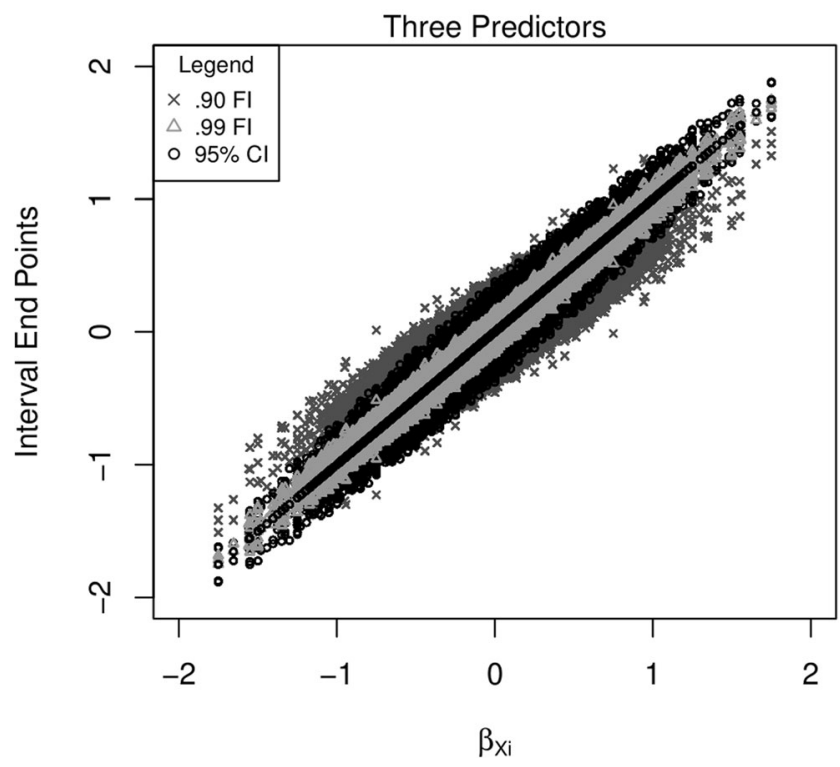

Fig. 5 End points of the confidence and fungible intervals for three predictors, plotted against the OLS point estimates, shown in black. Confidence intervals are for $N=100$. The values of $r_{\hat{y}_{\mathrm{a}} \hat{y}_{\mathrm{b}}}$ used here are .90 (dark gray) and .99 (light gray). Some interval end points associated with $r_{\hat{y}_{\mathrm{a}} \hat{y}_{\mathrm{b}}}=.90$, as well as for the $95 \%$ confidence intervals, overlap with the points for $r_{\hat{y}_{\mathrm{a}} \hat{y}_{\mathrm{b}}}=.99$

and $\beta_{X_{3}}$ are again identical). We use $r_{\hat{y}_{a} \hat{y}_{b}}=.99$ to minimize overlap of the points. Points are shaded according to whether both values of $\left|r_{X i * Y}\right|$ are equal to 0 or at least one is equal to $.1, .3$, or .5 , with darker points representing larger values. The minimum and maximum interval sizes for each $\beta$ were identical, and the largest intervals were $1.241,0.938$, and 0.441 , for $r_{\hat{y}_{a} \hat{y}_{b}}$ values of $.90, .95$, and .99 , respectively, and the smallest intervals were equal to zero-that is, no variability in the weights. For comparison, the $95 \%$ confidence intervals ranged in size from .048 to .757 . Fungible intervals of size zero occurred when all other predictors were uncorrelated with the criterion and the magnitudes of a given weight's fungible intervals were perfectly correlated across all three criterion values.

The fungible and confidence intervals again followed different patterns, similar to those observed in the twopredictor case. Larger values of $\beta$ are generally associated with tighter confidence intervals, but the fungible interval magnitudes are almost completely unrelated to the value of $\beta$. The confidence intervals again form a large cluster that slopes downward as the absolute value of $\beta$ increases, and the fungible intervals cluster in four overlapping groupings that are increasingly diffuse as the other correlations increase. There are four clusters 


\section{$\mathrm{Cl}$ and FI Interval Magnitude Three Predictors}

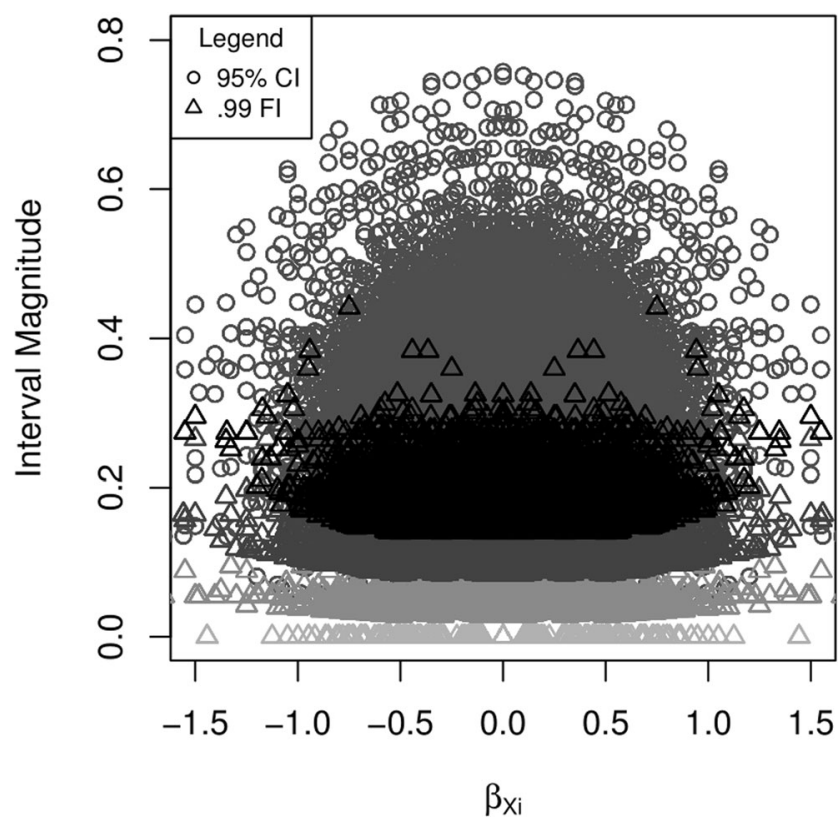

Fig. 6 Fungible interval (FI) $\left(r_{\hat{y}_{a}} \hat{y}_{b}=.99\right)$ and 95\% confidence interval (CI) magnitudes plotted against the value of $\beta_{X_{i}}$. Darker triangles indicate increasing values of at least one $\left|r_{X i * Y}\right|$. As can be seen, as the correlation magnitude increases, so too does the minimum magnitude for the FIs

here rather than six because there were only four correlation absolute values in this study (i.e., no |.2| or |.4|).

Another result of interest from this study is that the maximum range is much larger here than in the twopredictor case: 1.241 versus 0.453 for $r_{\hat{y}_{a} \hat{y}_{b}}=.90$. This difference occurs because the additional predictor affords more room for the weights to vary without strongly affecting the predicted values. The implication of this is that the more predictors there are in a regression model, the greater the potential parameter sensitivity and uncertainty regarding the correct values of individual effects. This holds true regardless of multicollinearity between the predictors.

Predictors of the fungible intervals While the fungible interval magnitude for the two-predictor case was very simply - and all but completely - explained by the magnitude of the other predictor's correlation with the criterion variable, $r_{X^{*} Y}$, an ellipse is a far more complicated shape than a line, so the other factors associated with shape and orientation (Jones, 2013) may have larger effects in this case. As a result, we again considered the following variables, in both pairs and trios, with interactions included: $\left|r_{X_{1} Y}\right|,\left|r_{X_{2} Y}\right|,\left|r_{X_{3} Y}\right|, R_{b}^{2}, V I F_{X_{1}}, V I F_{X_{2}}, V I F_{X_{3}}$; the determinant, condition number, and three eigenvalues of the predictor correlation matrix $\boldsymbol{R}_{\boldsymbol{x} x}$; the correlations between the predictor matrix eigenvectors and the OLS weights; as well as the three axes of the ellipsoid of all possible regressions given the predictor correlation matrix and a fixed $R_{\alpha}^{2}$.

The combination that yielded the most variance explained consisted of both values of $r_{X^{*} Y}$ (e.g., $\left|r_{X_{2} Y}\right|$ and $\left|r_{X_{3} Y}\right|$ for $\left.X_{1}\right)$ along with the third axis of the ellipsoid of all possible regressions for a given $R_{\alpha}^{2}$, as defined above. However, this combination of predictors yielded an $R_{b}^{2}$ value that was only .013 larger than the combination of the two values of $r_{X^{*} Y}$ and the value of $V I F_{X_{i}}$. The coefficient magnitudes were also similar, and the signs identical. Because the VIF is far more familiar to and easily understood by most researchers, as well as readily available in statistical software, we will discuss the model with the VIF instead of the third axis as the third predictor. The results of the regression using the third axis may be found in Appendix A. The highest observed $R_{b}^{2}$ values for other combinations of three predictors are also shown, along with the values for four and five predictors; of note is that $V I F_{X_{i}}$ consistently emerged as a strong explanatory variable. Our use of $V I F$ is also, of course, in keeping with the results of the two-predictor case, easing discussion. We will, however, return to this point in the General Discussion. Table 2 shows the results for $\beta_{X_{1}}$. Although the effects are again attributable to the values of $\left|r_{X_{i^{*}} Y}\right|$ in general, we explicitly reference each predictor in order to avoid confusion when discussing the interactions.

As in the previous study, the range of the fungible parameters increases with the magnitude of the correlations of the other predictors with the criterion variable. In this case, the other correlations, $\left|r_{X_{2} Y}\right|$ and $\left|r_{X_{3} Y}\right|$, are sufficient to yield $R_{b}^{2}=.778$, with all weights being positive. Including their interaction yields $R_{b}^{2}=.839$ and adding $V I F_{X_{1}}$ results in $R_{b}^{2}=.898$. Allowing for interactions between $V I F_{X_{1}}$ and the two correlations results in $R_{b}^{2}=.910$. Figure 7 displays the relationship between the fungible interval for $\beta_{X_{1}}$ as a function of $\left|r_{X_{2} Y}\right|$ and $\left|r_{X_{3} Y}\right|$, and like Fig. 4, it shows an increase in magnitude as the absolute correlation values of the other predictors increases. Interval magnitudes are symmetric across positive and negative correlation values.

This pattern, with a positive effect of the two single correlations between other predictors and the criterion and a negative interaction of the two correlations, means that together the two correlations have a disjunctive effect: It is sufficient for one of the two correlations to be large for the range to be large, as well. As in the twopredictor case, increasingly large correlations between the other predictors and the criterion variable allow for more variation in the weights without simply adding 
Table 2 Regression weights of explanatory variables for $\beta_{X_{1}}$ fungible intervals in the three-predictor case

\begin{tabular}{|c|c|c|c|c|}
\hline & \multicolumn{4}{|l|}{$r_{\hat{y}_{a} \hat{y}_{b}}$} \\
\hline & .90 & .95 & .99 & $\Delta R_{b}^{2}$ \\
\hline Intercept & 0.378 & 0.286 & 0.116 & \\
\hline$\left|r_{X_{2} Y}\right|$ & 0.111 & 0.084 & 0.040 & .379 \\
\hline$\left|r_{X_{3} Y}\right|$ & 0.111 & 0.084 & 0.040 & .399 \\
\hline$\left|r_{X_{2} Y}\right| \times\left|r_{X_{3} Y}\right|$ & $\begin{array}{l}- \\
0.0- \\
45\end{array}$ & $\begin{array}{l}- \\
0.0- \\
34\end{array}$ & $\begin{array}{c}- \\
0.0- \\
16\end{array}$ & .061 \\
\hline$V I F_{X_{1}}$ & 0.046 & 0.035 & 0.016 & .059 \\
\hline$\left|r_{X_{2} Y}\right| \times V I F_{X_{1}}$ & 0.011 & 0.008 & 0.004 & \\
\hline$\left|r_{X_{3} Y}\right| \times V I F_{X_{1}}$ & 0.011 & 0.008 & 0.004 & \\
\hline$\left|r_{X_{2} Y}\right| \times\left|r_{X_{3} Y}\right| \times V I F_{X_{1}}$ & $\begin{array}{c}- \\
0.0- \\
09\end{array}$ & $\begin{array}{l}- \\
0.0- \\
07\end{array}$ & $\begin{array}{l}- \\
0.0- \\
03\end{array}$ & $\begin{array}{l}.012 \\
R_{b}^{2}=.910\end{array}$ \\
\hline
\end{tabular}

Values of $\Delta R_{b}^{2}$ indicate increases in $R_{b}^{2}$ associated with each term being added to separate regression models. The intervals for the other predictors are similarly explained, with the predictor's VIF and the magnitudes of the other predictors' correlations. The regression weights are smaller in the columns farther to the right because the fungible intervals are smaller.

noise; that is, they compensate for the changes in weights, with collinear predictors providing a modest compensatory effect for the changes in weights.

\section{FI for Three Predictors}

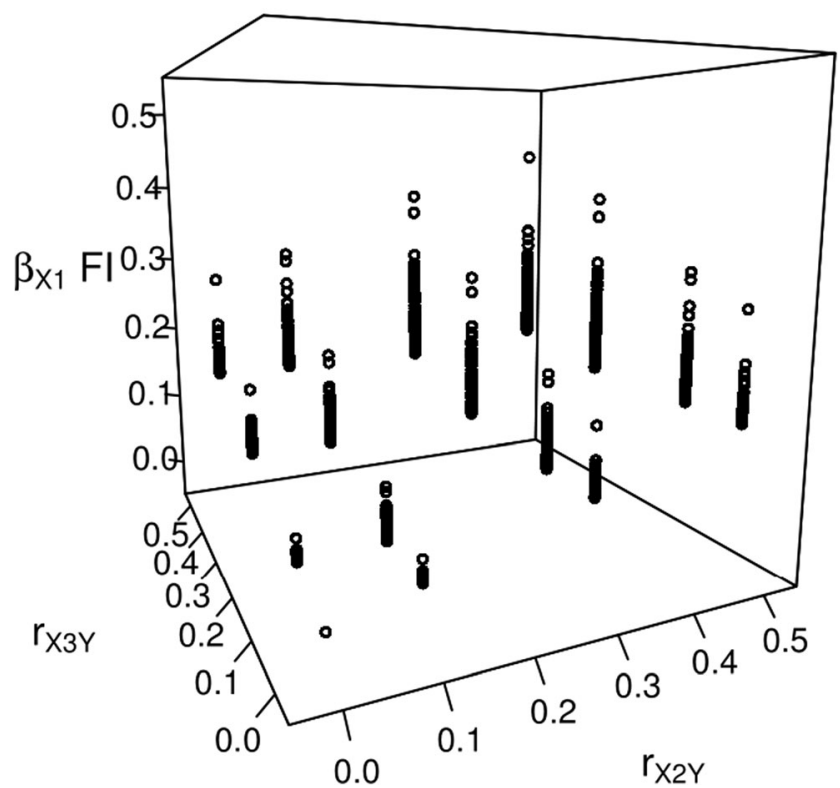

Fig. 7 Fungible interval (FI) magnitudes for $\beta_{X_{1}}$ and $r_{\hat{y}_{a}} \hat{y}_{b}=.99$, plotted in relation to $\left|r_{X_{2} Y}\right|$ and $\left|r_{X_{3} Y}\right|$

\section{Function to calculate fungible interval}

To facilitate the use of fungible intervals, we provide an $R$ ( $\mathrm{R}$ Core Team, 2018) function in Appendix B that is a wrapper function for Waller's (2008) original fungible function. The function accepts a predictor covariance matrix as input, $r x x$, and a vector of predictor-criterion covariances, rxy. If the input is a correlation matrix, then the reported weights will be standardized weights. Interactions must be calculated and included as separate variables in the covariance matrix. Allowing for the estimation of fungible weights with two predictors required a small modification to the original function, to allow two predictors; this is commented in the code. The calculation of fungible weights is otherwise unchanged (though there are some minor changes to the output provided by the function).

There are also two options to be made. The first is ryayb, which reflects the desired value of $r_{\hat{y}_{a} \hat{y}_{b}}$. This can be any value between 0 and 1, but an appropriate test of parameter sensitivity requires a relatively high value, and there is only one set of weights for values of 0 and 1 . The default value for ryayb is .99. The other option is sets, which is the number of desired fungible weight sets, with each set comprising one weight per predictor. This defaults to 1,000 . Smaller values will result in less precise fungible intervals. If there are only two predictors, then the two weight sets will be repeated and a smaller value - for example, 10 - can recover the fungible intervals without providing excessive and unnecessary repeated output.

The function outputs the minimum and maximum values of the fungible weights associated with each predictor, as well as the size of the interval. The OLS weights are also provided, for ease of comparison. Additionally, the values of $R_{b}^{2}$ and $R_{a}^{2}$ are provided, as well as the size of the difference between them. An example is provided in Appendix B.

\section{Discussion}

Our results show that the magnitude of the range of fungible weights for a given predictor, $X_{i}$, is primarily explained by the size of the correlation(s) between the other predictor variable(s) and the criterion variable, with multicollinearity contributing to the size by way of interactions with each $\left|r_{X_{*_{i}} Y}\right|$. Note that although multicollinearity did contribute to interval size, it did so far less than the predictorcriterion correlations, and no multicollinearity was necessary for an interval to be large. That the range is so simply explained affords a few straightforward points of discussion with high relevance for inferences made from regression analyses.

First, parameter sensitivity increases in cases when other predictors are highly correlated with the criterion 
variable, since they may compensate for any changes in a predictor's weight. To what degree this will impact a researcher's conclusions will depend, of course, on the sort of conclusions being drawn. If one wishes to draw inferences about relative predictor importance-for example, ranking - then large intervals are a concern, regardless of the value of a regression weight, particularly if the intervals overlap. However, if one is concerned only with sign and significance, then the importance of identifying sensitive weights increases as the regression weights decrease and the magnitudes of other correlations increase. This is because the fungible interval for a given predictor is increasingly likely to include near-zero weights, or even those of opposite sign. It is well-known that small effects are difficult to study because they tend to have lower power; a downwardly biased estimate will be even less likely to be statistically significant. Furthermore, in extreme cases, a biased estimate may be significant but have the wrong sign, as is implied by the example shown in Fig. 1.

Second, though we could not consider this in detail here, it seems that larger sets of predictors will lead to more sensitive weights, with each individual $\left|r_{X_{i^{*}} Y}\right|$ contributing less to the interval size. The maximum size of the fungible intervals was significantly higher in the three-predictor than in the twopredictor case (1.241 vs. 0.453 ), and whereas for two predictors $\left|r_{X_{i^{*}} Y}\right|$ was sufficient to explain $99 \%$ of the variation, for three predictors, each $\left|r_{X^{*} Y}\right|$ only explained about $39 \%$, for a total of $78 \%$. A negative interaction between these two variables explained another $6 \%$, for a total of $84 \%$. This suggests that increasing the number of predictors results in a cumulative effect on interval size by way of increasingly smaller and nonlinear effects of each individual $\left|r_{X_{i^{*}} Y}\right|$ on interval size. A perfect disjunctive relationship would mean that one other predictor with a high correlation would be sufficient. Because researchers generally use more than three predictors (e.g., control variables), it will be important for future research to explore the behavior of fungible intervals in relation to the number of predictors.

Third, as is to be expected, predictor multicollinearity results in less trustworthy weights. This effect is, however, surprisingly small here, and it is not necessary that any multicollinearity be present for the weights to be sensitive. It does appear, however, that the effects of multicollinearity may increase with the number of predictors. In the two-predictor case, the effects of VIF only explained $1 \%$ of the variance beyond $\left|r_{X_{*} Y}\right|$, but in the three-predictor case, it explained an additional $7 \%$ of the variance. It is also worth noting that although VIF is both familiar and readily available, for the three-predictor case the third, smallest axis of the allpossible-regressions ellipsoid explained $1 \%$ more variance than $V I F$ in the three-predictor case (VIF was nonetheless a consistently strong explanatory variable; see Appendix A). It is difficult to extrapolate to a larger number of predictors based on only two sets of results, but given that there are only two weight sets for the geometrically simple two-predictor case, but an infinite number for the more complex cases of three or more predictors, it seems that the smallest axis of the (hyper) ellipsoid may be a better predictor of interval size for three or more predictors. Whether or not this is the case will require additional research, but it appears that in practice, measures of multicollinearity are largely substitutable with respect to fungible interval size (see Appendix A).

Finally, our results are of note for mediation models (where the total effect of a predictor variable $X$ on a criterion variable $Y$ is decomposed into a direct effect and an indirect effect involving a mediator $M$; Hayes, 2013). If the correlations between $X$ and $Y$ and $M$ and $Y$ are both high, then it follows that the corresponding regression weights used for the direct and indirect effects will be sensitive. Mediation models are also unusual in that multicollinearity is to some degree desirable, as a larger correlation between $X$ and $M$ will increase the size of the indirect effect but also result in a larger value of VIF. Estimated mediation effects are then likely to be particularly sensitive (cf. Sobel, 2008).

A limitation of our study is that we exclusively focused on the range of fungible weights per predictor. Although this simplification allowed us to illustrate the differences relative to confidence intervals and to derive some simple and clear conclusions, a summary of sensitivity that jointly considers all predictors (e.g., the area encompassed by the ellipse) might be of greater interest in other contexts, because it would provide a global summary of parameter sensitivity for all predictors considered jointly. Additionally, since we did not consider four or more predictors here, we were unable to test whether the smallest axis of the ellipsoid is a better predictor than VIF in general, nor could we explore how much the variance explained by each $\left|r_{X_{*_{i} Y}}\right|$ drops as the number of predictors increases.

\section{Conclusion}

Although users of regression are aware that in some sense their models may be inaccurate and that the estimates that an analysis yields may well be biased, it is difficult to know the nature of such bias, as well as any potential effects on the trustworthiness of the effects. It follows, then, that it is difficult to identify in advance the 
conditions under which the estimated parameters will be sensitive due to individual violations - that is, be less trustworthy, in the sense of less valid (Green, 1977). However, our results here suggest that it is not necessary to have knowledge of specific model violations in order to identify situations in which the parameters of interest will be sensitive. The potential consequences can be assessed in terms of an informative fungible interval for different values of the prespecified correlation, without assuming a specific type of model violation. Knowledge of the specific violation would, of course, give more specific indications than is possible with a fungible interval, but such knowledge is commonly not available, and if it were, then the violation could be remedied directly.

Finally, we hope that the function we provide will ease exploring parameter sensitivity for users of regression analysis. Although the fungible intervals should not be used for null hypothesis testing of regression weights, they are still useful as indications of the confidence one may have in the weights, from the viewpoint of model validity.

Author note This study was not preregistered and makes use of no data. However, code for the simulations and analyses is available upon request to the corresponding author.

Table 3 Regression weights of explanatory variables for $\beta_{X_{1}}$ fungible intervals in the three-predictor case using the third axis of the ellipsoid, $l_{3}$

\begin{tabular}{lcccc}
\hline & $r_{\hat{y}_{a} \hat{y}_{b}}$ & & & \\
\cline { 2 - 5 } & .90 & .95 & .99 & $\Delta R_{b}^{2}$ \\
\hline Intercept & 0.359 & 0.272 & 0.128 & \\
$\left|r_{X_{2} Y}\right|$ & 0.092 & 0.070 & 0.033 & .379 \\
$\left|r_{X_{3} Y}\right|$ & 0.092 & 0.070 & 0.033 & .399 \\
$\left|r_{X_{2} Y}\right| *\left|r_{X_{3} Y}\right|$ & - & - & - & .061 \\
& $0.0-$ & $0.0-$ & $0.0-$ & \\
$l_{3}$ & -51 & 39 & 18 & .059 \\
& $0.0-$ & 0.043 & 0.020 & \\
$\left|r_{X_{2} Y}\right| * l_{3}$ & 0.022 & 0.017 & 0.008 & \\
$\left|r_{X_{3} Y}\right| * l_{3}$ & 0.022 & 0.017 & 0.008 & \\
$\left|r_{X_{2} Y}\right| *\left|r_{X_{3} Y}\right| * l_{3}$ & - & - & - & .025 \\
& $0.0-$ & $0.0-$ & $0.0-$ & $R_{b}^{2}=.923$ \\
& 01 & 01 & 01 & \\
\hline
\end{tabular}

Values of $\Delta R_{b}^{2}$ indicate increase in $R_{b}^{2}$ associated with each term added to separate regression models. The results for other variable intervals were the same. The regression weights are smaller in the columns farther to the right because the fungible intervals are smaller.
Table 4 Largest $R_{b}^{2}$ values yielded by three, four, and five explanatory variables for fungible intervals

\begin{tabular}{llllll}
\hline$\lambda_{1}$ & .870 & $V I F_{X i} * l_{2}$ & .937 & $V I F_{X i} * l_{1} * l_{3}$ & .947 \\
$\lambda_{3}$ & .897 & $l_{3} * \lambda_{3}$ & .938 & $V I F_{X i} * l_{2} * \lambda_{3}$ & .948 \\
$V I F_{X i}$ & .910 & $V I F_{X i} * l_{3}$ & .938 & $V I F_{X i} * l_{3} * \kappa$ & .948 \\
$\kappa$ & .914 & $l_{3} * \kappa$ & .942 & $V I F_{X i} * l_{2} * \kappa$ & .949 \\
$l_{3}$ & .923 & $l_{1} * l_{3}$ & .944 & $V I F_{X i} * l_{1} * \kappa$ & .950 \\
\hline
\end{tabular}

These $R_{b}^{2}$ values are yielded by the interaction between the two values of $\left|r_{X i * Y}\right|$ and different variables related to multicollinearity and the shape of the all possible regressions ellipsoid. $\lambda_{i}$ denotes the eigenvalues of the predictor matrix, $l_{i}$ the axes of the ellipsoid, and $\kappa$ the condition number, $\lambda_{\max } / \lambda_{\min }$. The $R_{b}^{2}$ from the correlations alone was .839 .

\section{Appendix A}

In the body of the text we reported the results of the regression using the two values of $\left|r_{X_{i^{*}} Y}\right|$ and $V I F_{X i}\left(R_{b}^{2}=.910\right)$, because it yielded a value of $R_{b}^{2}$ comparable to that of the regression using the two values of the two values of $\left|r_{X_{i^{*}} Y}\right|$ and the third axis of the ellipsoid, $l_{3}\left(R_{b}^{2}=.923\right)$. Doing so facilitated our general point that the size of the fungible interval is explained by the values of $\left|r_{X^{*} Y}\right|$ and multicollinearity (more specifically, by the shape of the all-possible-regressions ellipsoid defined by $R_{a}^{2}=\boldsymbol{a}^{\prime} \boldsymbol{R}_{x x} \boldsymbol{a}$ ), but we report the effects of $l_{3}$ here for completeness. These results are shown in Table 3. The effects of $V I F_{X i}$ and $l_{3}$ are comparable in size, with the effects of $l_{3}$ being slightly stronger. All weights were of the same sign in both regression analyses. All explanatory variables were standardized in order to make the effects comparable within the analyses, without masking differences between the values of $r_{\hat{y}} \hat{y}_{b}$.

As an additional check on our results and decision to focus on $V I F_{X i}$, we also considered other combinations of three to five explanatory variables, focusing on other properties of the (hyper)ellipsoid. Specifically, we considered the effects of $\left|r_{X_{1} Y}\right|,\left|r_{X_{2} Y}\right|,\left|r_{X_{3} Y}\right|, R_{b}^{2}, V I F_{X_{1}}, V I F_{X_{2}}, V I F_{X_{3}}$; the determinant, condition number, and three eigenvalues of the predictor correlation matrix; the correlations between the predictor matrix eigenvectors and the OLS weights; and the three axes of the ellipsoid. All variables were allowed to interact.

Table 4 shows the five highest values of $R_{b}^{2}$ observed for each number of variables. As can be seen, $V I F_{X i}$ consistently emerged as one of the best predictors of interval size. Additionally, for five explanatory variables, the five combinations with the highest values of $R_{b}^{2}$ all included $V I F_{X i}$. Taken together, then, $V I F_{X i}$ can be considered a good and convenient indicator of interval size, but full consideration of the size of the interval would require also examining the other indicators of the shape of the (hyper)ellipsoid. 


\section{Appendix B}

\section{\#\#\#\#\#}

\#\# Fungible interval wrapper for Fungible function from Waller (2008)

\#\# Wrapper written by Robert Agler, with the following alterations to the original function

\#\# removed all text printed during the calculation of weights

\#\# changed colnames for a.matrix to match input column names

\#\# changed code involved in calculation of $\mathrm{d}$ so as to allow just two predictors

\#\# June 21, 2018

\#\#

\#\# rxx = matrix of predictor (co)variances

\#\# rxy $=$ vector of predictor-criterion covariances

\#\# sets = number of fungible weight sets to calculate

\#\# ryayb = desired correlation between OLS predictions and fungible predictions

fungibleInterval $<$ - function $(\mathrm{rxx}$, rxy, sets $=1000$, ryayb $=.99)\{$

fungInt $<$ - function $(x) \operatorname{return}(\mathrm{c}(\operatorname{range}(\mathrm{x}), \operatorname{range}(\mathrm{x})[2]-\operatorname{range}(\mathrm{x})[1]))$

Fungible $<$ - function(R.X,rxy,r.yhata.yhatb $=$ ryayb, sets=nsets $)\{$

GenU $<$ - function(mat,u) \{

\#\# Generate U matrix via Gram Schmidt

$\mathrm{p}<-\operatorname{ncol}(\mathrm{mat})$ 


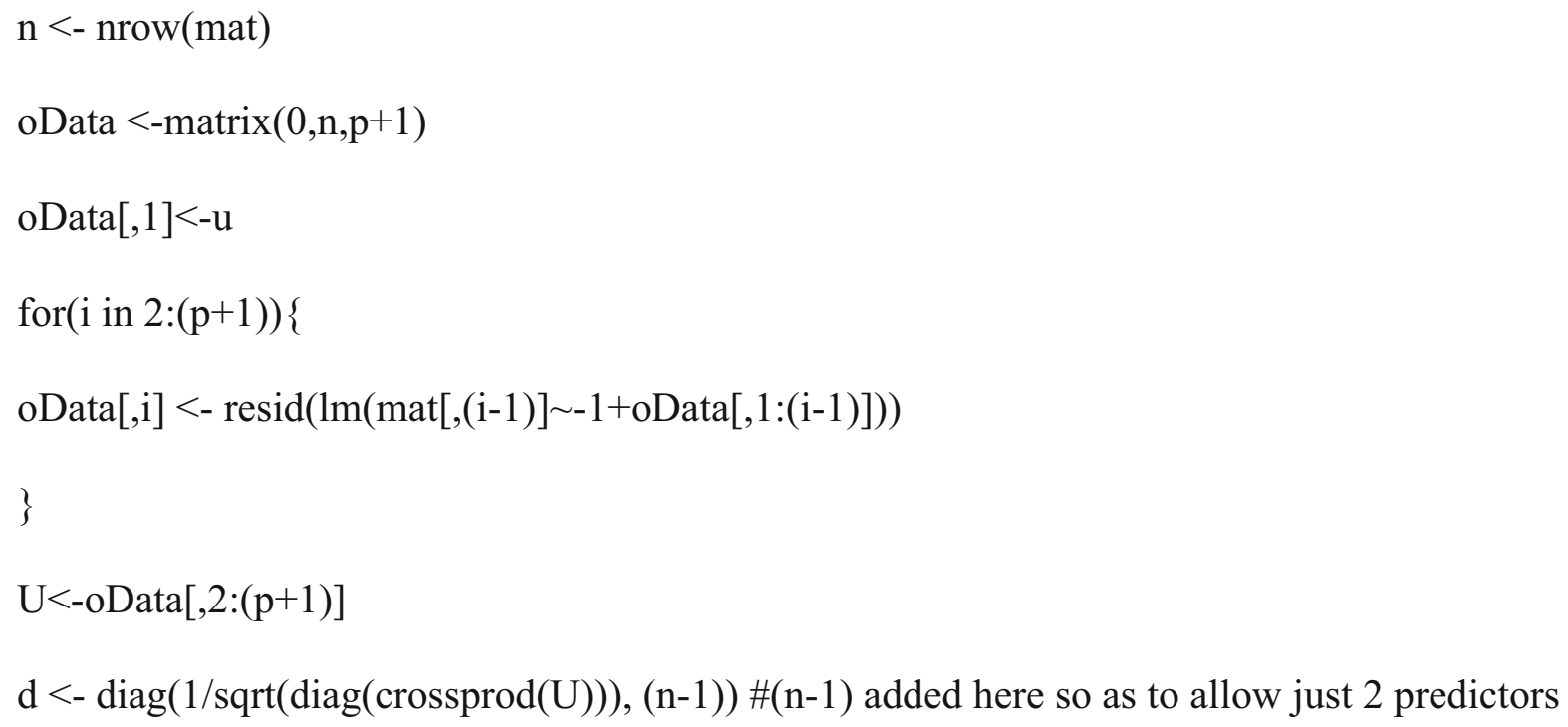




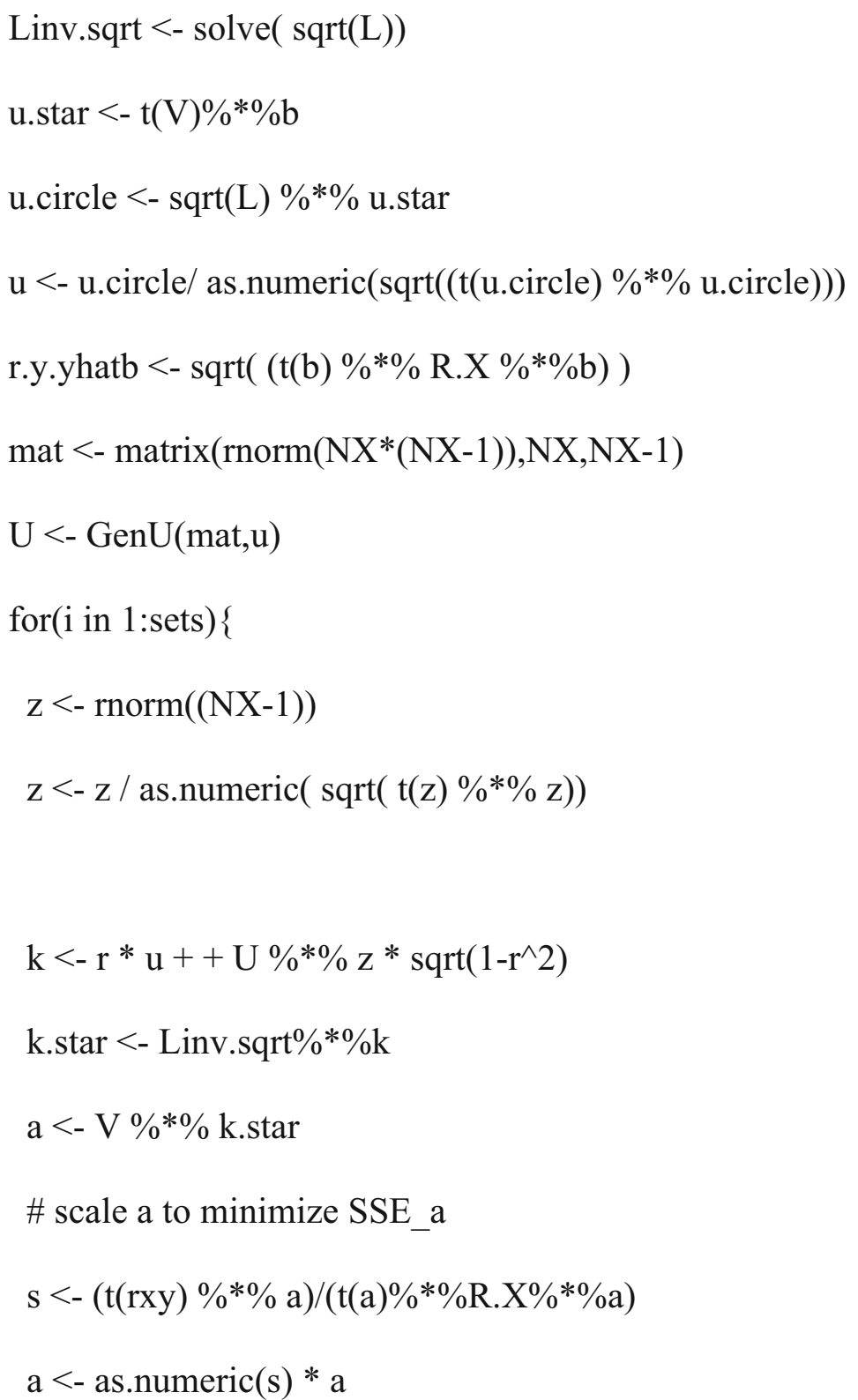

as.numeric(s) * a

a.matrix $[\mathrm{i}]<,-\mathrm{a}$

k.matrix $[\mathrm{i}]<,-\mathrm{k}$

\} 
colnames(a.matrix) $<-$ colnames $($ data $)[x]$

\# Compute Expected Moments

$\mathrm{G}<-\mathrm{V} \% * \%$ Linv.sqrt $\% * 0 \mathrm{U}$

esq $<-\left(1-\mathrm{r}^{\wedge} 2\right)$

\# Expected a

mn.a $<-\mathrm{r}^{\wedge} 2 * \mathrm{~b}$

Ezsq $<-1 /(N X-1)$

\# Expected covariance matrix

cov.a $<-$ as.numeric $\left(\mathrm{r}^{\wedge} 2 *\right.$ r.y.yhatb^2)* Ezsq $*$ esq $* \mathrm{G} \% * \% \mathrm{t}(\mathrm{G})$

Dmat $<-\operatorname{diag}(1 / \operatorname{sqrt}(\operatorname{diag}(\operatorname{cov} . \mathrm{a})))$

cor.a $<$ - Dmat $\% * \%$ cov.a $\% * \%$ Dmat

$\operatorname{list}(a=a \cdot m a t r i x, k=k \cdot$ matrix $, b=b, u=u, r . y h a t a \cdot y h a t b=r . y h a t a \cdot y h a t b, r . y . y h a t b=r . y \cdot y h a t b$, $\operatorname{cov} . a=\operatorname{cov} . a$, cor $. a=$ cor.$a)$

\}

fung $<-$ Fungible(R.X = rxx, rxy = rxy, r.yhata.yhatb $=$ ryayb, sets $=$ sets $)$

weights $<-\mathrm{t}($ apply (fung\$a, 2, FUN = fungInt)) 
weights $<$ - cbind(fung\$b, weights)

colnames(weights) <- c("OLS weight", "FI Min", "FI Max", "FI Size")

r2.ols <- as.numeric(fung $\$ r$.y.yhatb) $)^{\wedge} 2$

r2.fung $<-\left(\right.$ fung $\$$ r.y.yhatb $*$ ryayb) ${ }^{\wedge} 2$

r2 <- matrix(c(r2.ols, r2.fung, r2.ols-r2.fung), 1, 3, dimnames = list(1, c("R2.ols", "R2.fung", "Difference")))

out $<$ - list $($ weights $=$ weights,

$$
\mathrm{r} 2=\mathrm{r} 2 \text { ) }
$$

return(out)

\}

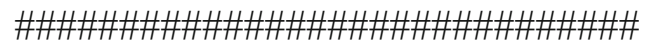

\#\#\# Example

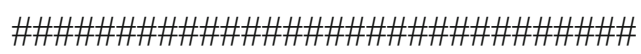

true.cors <- matrix(.3, 6, 6, dimnames = list(c("x1", "x2", "x3", "x4", "x5", "y"), c("x1", "x2", "x3", "x4", "x5", "y")))

$\operatorname{diag}($ true.cors $)<-1$

true.cors $[, 6]<-$ true.cors $[6]<,-\mathrm{c}(.1, .2, .3, .4, .5,1)$ 
intervals $<$ - fungibleInterval(true.cors[1:5, 1:5], true.cors[1:5, 6])

intervals

\$'weights'

OLS weight FI Min FI Max FI Size

$\begin{array}{lllll}x 1 & -0.149350649 & -0.23447761 & -0.05938791 & 0.1750897\end{array}$

$\begin{array}{llllll}x 2 & -0.006493506 & -0.09746386 & 0.08361164 & 0.1810755\end{array}$

$\begin{array}{llllll}x 3 & 0.136363636 & 0.04555698 & 0.22158004 & 0.1760231\end{array}$

$\begin{array}{llllll}\times 4 & 0.279220779 & 0.19294568 & 0.35616610 & 0.1632204\end{array}$

$\begin{array}{lllll}\times 5 & 0.422077922 & 0.34525217 & 0.48301010 & 0.1377579\end{array}$

$\$ r 2$

R2.01s R2.fung Difference

$10.3474026 \quad 0.3404893 \quad 0.006913312$

\section{References}

Box, G. E. (1976). Science and statistics. Journal of the American Statistical Association, 71, 791-799.

Edwards, M. C. (2013). Purple unicorns, true models, and other things I've never seen. Measurement: Interdisciplinary Research and Perspectives, 11, 107-111.

Green, B. F. (1977). Parameter sensitivity in multivariate methods. Multivariate Behavioral Research, 12, 263-287.

Hayes, A. F. (2013). Introduction to mediation, moderation, and conditional process analysis: A regression-based approach. New York, NY: Guilford Press.

Jaccard, J., \& Wan, C. K. (1995). Measurement error in the analysis of interaction effects between continuous predictors using multiple regression: Multiple indicator and structural equation approaches. Psychological Bulletin, 117, 348-357.

Jones, J. A. (2013). Fungible weights in logistic regression (Unpublished doctoral dissertation). University of Minnesota, Minneapolis, MN.

Jones, J. A., \& Waller, N. G. (2016). Fungible weights in logistic regression. Psychological Methods, 21, 241-260.

Lee, T., MacCallum, R. C., \& Browne, M. W. (2018). Fungible parameter estimates in structural equation modeling. Psychological Methods, 23, 58-75. doi:https://doi.org/10.1037/met0000130
Pek, J., Chalmers, R. P., \& Monette, G. (2016). On the relationship between confidence sets and exchangeable weights in multiple linear regression. Multivariate Behavioral Research, 51, 719-739.

R Core Team (2018). R: A language and environment for statistical computing. Vienna, Austria: R Foundation for Statistical Computing. Retrieved from http://www.R-project.org/

Sobel, M. E. (2008). Identification of causal parameters in randomized studies with mediating variables. Journal of Educational and Behavioral Statistics, 33, 230-251.

Waller, N. G. (2008). Fungible weights in multiple regression. Psychometrika, 73, 691-703.

Waller, N. G., \& Jones, J. A. (2009). Locating the extrema of fungible regression weights. Psychometrika, 74, 589-602.

Waller, N., \& Jones, J. (2011). Investigating the performance of alternate regression weights by studying all possible criteria in regression models with a fixed set of predictors. Psychometrika, 76, 410-439.

Publisher's note Springer Nature remains neutral with regard to jurisdictional claims in published maps and institutional affiliations. 